\title{
Assessing the thermal comfort effects of green spaces: a systematic review of methods, parameters, and plants attributes
}

\begin{abstract}
The expansion of urbanization leads to increases in urban populations and manmade constructions. Likewise, problems like changing local climates negatively affect urban heat islands and human outdoor thermal comfort. This paper is based on recent studies on the effects of green spaces and plants on the microclimate and thermal comfort of recent years. The topics studied included physical properties of plants, location and vegetation cover, planting densities and crown density, plant element, leaf type, planting patterns and arrangement, and Albedo. The results of the review revealed that several factors with effects on the cooling effect of plants have not received adequate attention in previous studies yet. These include the effects of patterns, arrangement of various plant species, different tree forms and shrubs, the distribution and connection of green space patches in the landscape, the direction of the planting rows in different wind conditions, among others. By the end of this review, suggestions could be made about what possible future studies could undertake to do by way of adopting more precise and comprehensive approaches to different characteristics of plants, different landscape patterns, and the effects of different arrangements of elements in terms of microclimate improvement.
\end{abstract}

Keyword: Climate change; Urban heat Island; Outdoor thermal comfort; Urban green space; Cooling effect; Vegetation characteristics; Planting pattern 